\title{
Characteristics and Outcomes of Breast Cancer- Retrospective Analysis from a Tertiary Center in India.
}

\author{
Vidhya $\mathbf{R}^{1}$, Kalaichelvi $\mathrm{K}^{2}$, Suresh Kumar $\mathbf{S}^{3}$, Raja $\mathbf{G}^{3}$, Karthik $\mathbf{R}^{4}$ \\ 'DM Resident, Department of Medical Oncology, Madras medical College, Chennai, Tamil Nadu, ${ }^{2}$ Professor \& HOD, Department of Medical Oncology, Madras \\ medical College, Chennai, Tamil Nadu, ${ }^{3}$ Asst Professor, Department of Medical Oncology, Madras medical College, Chennai, Tamil Nadu, ${ }^{5}$ Research Scientist, \\ Multidisciplinary Research Unit, Madras medical College, Chennai, Tamil Nadu, India.
}

\section{Abstract}

Background: Breast cancer is the most common cancer among women in India and globally and the leading cause of cancer mortality among females. In India, the locally advanced breast cancer (LABC) constitutes 50 to $60 \%$ of the patients with Luminal A and Luminal B subtype, $16.7 \%$ HER2neu positive and 29.8\% triple negative. Subjects and Methods: Breast cancer treated in the Department of Medical Oncology, RGGGH, Chennai from January 2014 to December 2016 were included in the study. Six hundred and twelve early and LABC patients were analyzed. Results: Among 612 patients, 315 (42.8\%) were had early breast cancer and 297 (40.4\%) were had locally advanced breast cancer. Among them 30.7\% luminal subtype, $11.4 \%$ triple positive, $13.7 \%$ HER2 positive, $25.6 \%$ triple negative and $17.5 \%$ had unknown status. The overall mean DFS and OS in early breast cancer was 57 months and 58 months respectively and locally advanced breast cancer was 51months and 52.5 months respectively. Discussion: Prevalence of TNBC in India is considerably higher compared with Western populations with Indian trial having $51.2 \%$ HR positive tumors, HER2 positive tumors in $16.7 \%$ and TNBC in $29.8 \%$. In our study, 30.7\% Luminal subtype, $11.4 \%$ triple positive, $13.7 \%$ HER 2 positive, $25.6 \%$ triple negative and $17.5 \%$ had unknown status. The overall mean DFS and OS in early breast cancer was 57 months and 58 months respectively and locally advanced breast cancer was 51 months and 52.5 months respectively. Conclusion: In our study we found that most of our patients present with advanced disease and TNBC and HER2 positive disease compared to western countries. In our study we found the survival of our patients was comparable with our Indian survival data, but less than western patients.

Keywords: Breast Cancer, Baseline Characters, Molecular Subtypes.

Corresponding Author: Dr. Vidhya R, DM Resident, Department of Medical Oncology, Madras medical College, Chennai, Tamil Nadu, India.

Received: June 2019

Accepted: June 2019

\section{Introduction}

In 2018, 2.1 million women globally were diagnosed to have breast cancer and this is the most common cancer among women in India and globally. It is also the leading cause of cancer mortality among females. Genetic predisposition accounts for 5-10\% of breast cancer. In India the incidence of breast cancer is in rising trends due to changes in reproductive risk factors, dietary habits and lifestyle changes. ${ }^{[1]}$

A study from north India in women has reported a strong association of risk factors like breast-feeding, location (urban/rural) and increased BMI with breast cancer. ${ }^{[2]}$ Increased breastfeeding and physical activity were protective for both ER+ and ER- breast cancer. ${ }^{[3]}$ Lifetime duration of breastfeeding was inversely associated with breast cancer risk among premenopausal women. ${ }^{[4]}$ Living in rural areas decreases the risk for breast carcinoma as compared to urban counterparts mainly due their adherence to rural lifestyle. ${ }^{[5]}$

According to globacon 2018, the higher incidence of breast cancer occurs in Australia/ New Zealand (94.2/ 100,000) and lowest in South Central Asia (25.9/100,000). In India age standardized ratio is $24.7 / 100,000 .^{[6]}$

The peak incidence of breast cancer occurs between 60-64 years in western countries but in Indian women it is between 45-49years.7 Early stage breast cancer (>60\%) is common in the western population with $72.7 \%$ Luminal A sub type, $12.2 \%$ TNBC , 15-25\% HER2 positive and $12 \%$ unknown status. ${ }^{[8,9]}$

How lader et al reported best survival pattern among women with $\mathrm{HR}+/ \mathrm{HER} 2$ - subtype (survival rate of $92.5 \%$ at 4 years), followed by HR+/HER2+ (90.3\%), HR-/HER2+ $(82.7 \%)$, and finally worst survival for triple-negative subtype $(77.0 \%){ }^{[10]}$

In India, the locally advanced breast cancer (LABC) constitutes 50 to $70 \%$ of the patients presenting for treatment which includes 6-25\% having distant metastases with 51.2\%Luminal A and Luminal B subtype, $16.7 \%$ HER2neu positive disease and $29.8 \%$ triple negative. ${ }^{[1,12]}$ The 5 year disease free survival (DFS) rate of $70 \%$ and overall survival rate of $78 \%$ is seen for early breast cancer. ${ }^{[13]}$ 


\section{Subjects and Methods}

Cases of early and locally advanced breast cancer which were treated in the Department of Medical Oncology, from January 2014 to December 2016 were included in the study. Six hundred and twelve patients were eligible for analysis. Retrospective review all available medical records to obtain information of patient characteristics, clinical presentation, TNM stage, pathology and receptor details, treatments, recurrence, survival and follow-up was done. Last follow up was till March 2019.

\section{Statistical methods}

The data was analysed with IBM.SPSS statistics software version 20.0. To describe about the data descriptive statistics, frequency analysis and percentage analysis was used for categorical variables and Kaplan meier survival curve used for comparing groups.

\section{Results}

Among 736 breast cancer individuals 612 with early and locally advanced breast cancer individuals were analyzed [Figure1]. Three hundred and fifteen (42.8\%) had early breast cancer and 297 (40.4\%) had locally advanced breast cancer [Figure1].

The peak incidence occurred between 41-50 years of age(ranges between 24-84 years) [Figure2] with $42 \%$ in premenopausal and $58 \%$ in postmenopausal status.

\section{Stage}

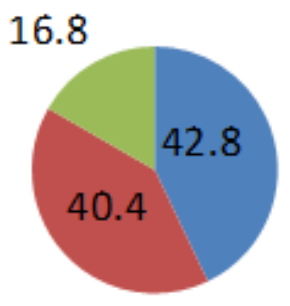

Early

LABC

Metastatic

Figure 1: Stage distribution.

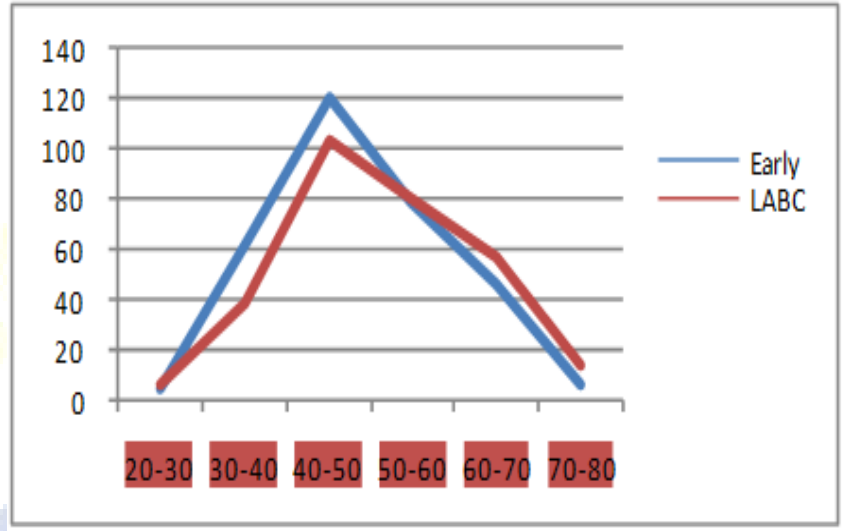

Figure 2: Age distribution.

Table 1:Hormonal receptor status.

\begin{tabular}{|l|l|l|l|l|l|l|}
\hline S.No & Stage & HR +ve & Triple +ve & HER2 +ve & TNBC & Unknown status \\
\hline 1. & Early & $33.3 \%$ & $9.5 \%$ & $12.4 \%$ & $33 \%$ & $11.4 \%$ \\
\hline 2. & LABC & $28 \%$ & $13.5 \%$ & $15.2 \%$ & $17.8 \%$ & $23.9 \%$ \\
\hline 3. & Total & $30.7 \%$ & $11.4 \%$ & $13.7 \%$ & $25.6 \%$ & $17.5 \%$ \\
\hline
\end{tabular}

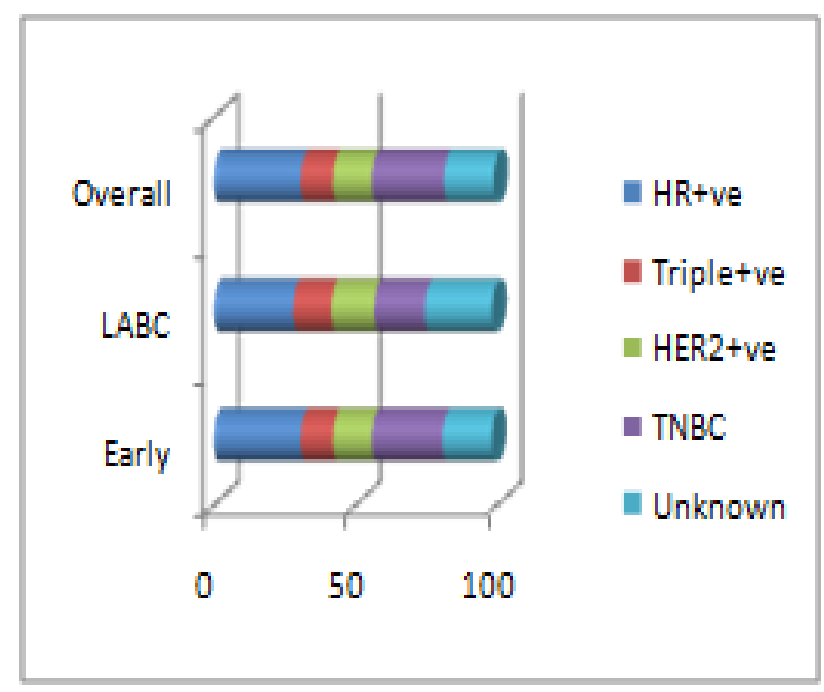

Figure 3: Hormonal receptor status

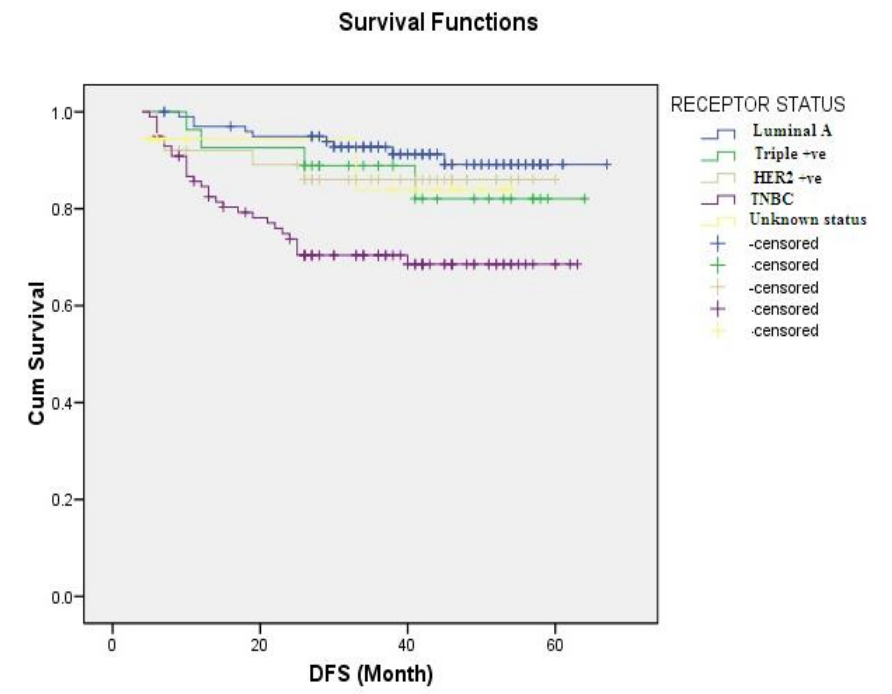

Figure 4. DFS in Early breast cancer 


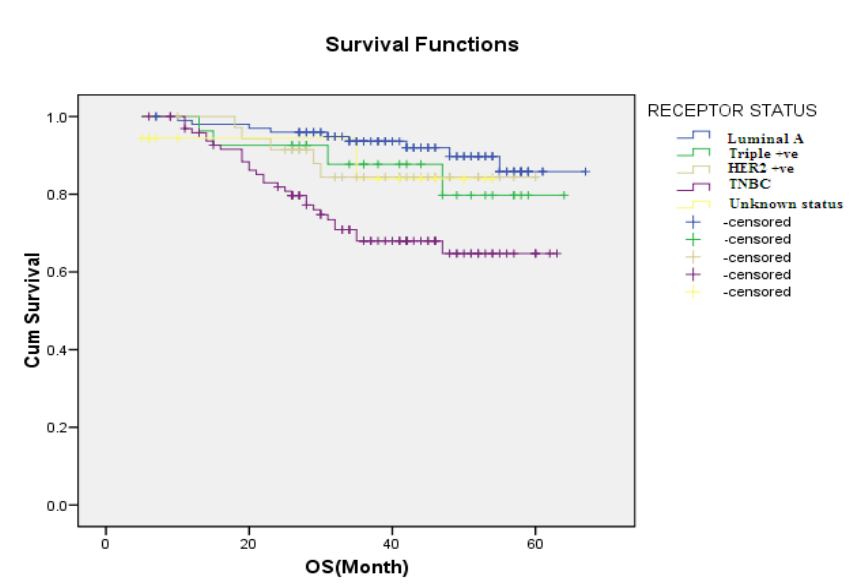

Figure 5: OS in Early breast cancer

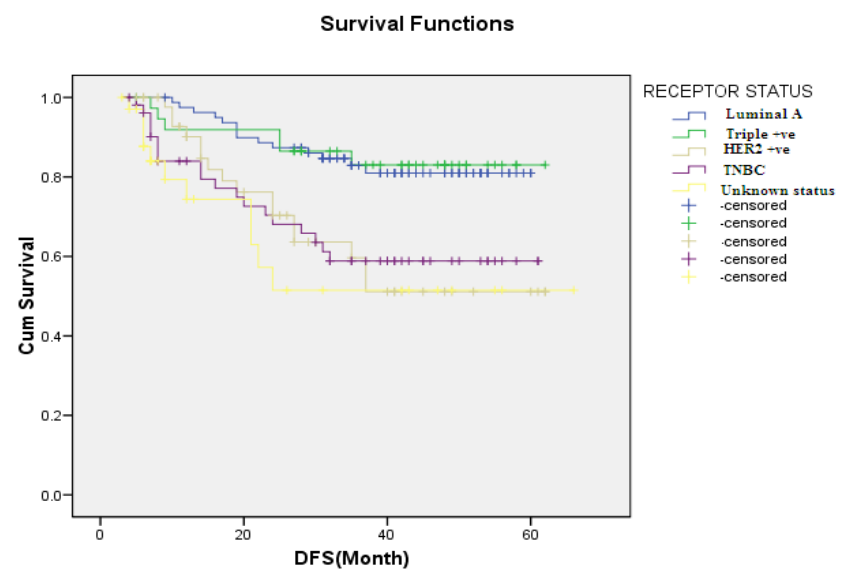

Figure 6: DFS in LABC

All the patients received standard therapy with combination of surgery, chemotherapy and radiotherapy followed by hormonal therapy (eligible patients).Thirteen percentage of women defaulted treatment with highest being in LABC $(15.2 \%)$ followed by early breast cancer $(9.8 \%)$.

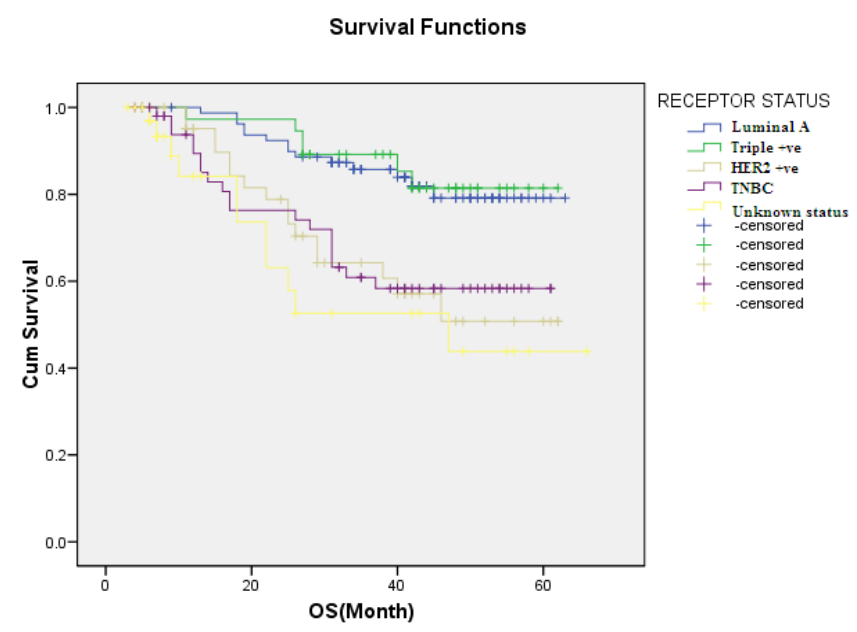

Figure 7: OS in LABC

At the median follow up of 45 months (28-64 months), 48 $(15.2 \%)$ patients in early breast cancer and $65(21.9 \%)$ patients in LABC died. The overall mean DFS and OS in early breast cancer was 57 months and 58 months respectively and in locally advanced breast cancer was 51months and 52.5 months respectively. In early breast cancer luminal A had DFS and OS of $86 \%$ and $90 \%$ compared to TNBC with $70 \%$ and $60 \%$ respectively and patients with LABC luminal A had DFS and OS of $82 \%$ and $80 \%$ compared to TNBC with $60 \%$ and $58 \%$ respectively as shown in [Figure 4-7].

\section{Discussion}

The aim of this retrospective analysis was to study the baseline characteristics and outcomes of early and locally advanced breast cancer patients who were treated from January 2014 to December 2016 at a tertiary care center in India.

The incidence of breast cancer is increasing rapidly in Asian countries and being the most frequently diagnosed cancer and the leading cause of cancer death in women. ${ }^{[14]}$

According to Globocan 2018, breast cancer is the most common cancer which constitutes $24.2 \%$ among all cancers and most common cause of mortality (15\%) among females. The breast cancer is the most frequently diagnosed cancer in the vast majority of the countries (154 of 185) and is also the leading cause of cancer death in over 100 countries and in India breast cancer in females constitutes $27.7 \%$ among all cancers(overall 14\%) with mortality of $12.1 \% .^{[6,15]}$

Leong et al reported the incidence of early breast cancer in western countries ranging from $60 \%$ to $70 \%$ and the remaining being advanced breast cancers. ${ }^{[16]}$

Women with breast cancer in India are detected clinically in contrast to western countries where most breast cancers are detected by screening. Up to two-thirds of patients present with local invasion with one-third being with skin and/or chest wall involvement (T4a-c) and 6-25\% present with metastases. $^{[11]}$

In this study $40.4 \%$ had early breast cancer, $42.8 \%$ had LABC and $16.8 \%$ had metastatic disease at presentation. Ten year age distribution peaks between 41-50 years in early and advanced breast cancer (LABC) with $42 \%$ were being premenopausal. The median age of patients (49 years) is approximately a decade younger than the West. ${ }^{[17]}$ This is likely to be due to the different age distribution of the Indian population, where only $7 \%$ of the population is above the age of 60 years. ${ }^{[18]}$

The proportion of tumors with various patterns of receptor expression is reported in the Western literature. ${ }^{[19,20]}$ Nadia et al reported US incidence of breast cancer subtypes as Luminal A 72.7\%, Luminal B 10.3\%, and TNBC 12.2\%, HER2 positive $15-25 \%$ and $12 \%$ unknown status. (8) Saber Fallahpour et al reported as luminal A subtype was the most commonly diagnosed, accounting for $59.0 \%$ of all cases, with a rate of 103.3 per 100000 , followed by triplenegative (15.1 per 100 000), luminal B (13.5 per 100 000) and HER2-enriched (7.0 per 100 000) with survival differed significantly between each molecular subtype, with patients with the luminal A subtype experiencing the longest 
survival, followed by those with the luminal B and HER2enriched subtypes. The poorest survival was observed among patients with the triple-negative subtype. ${ }^{[21]}$

Mustapha Abubakar reported , 34\% were luminal A-like, $33 \%$ were luminal B-like, $13 \%$ were HER2-enriched, and $20 \%$ were triple-negative, respectively with overall, allcause mortality and recurrence differed significantly by tumor subtype. In general, women with luminal A-like tumors had better survival outcomes than those with the other subtypes. ${ }^{[22]}$

A tertiary care centre in south India has recently reported a high percentage of triple negativity $(25 \%)$ in their breast cancer patients (compared to the West) and a similar percentage of patients (27\%) with HER2 positivity. ${ }^{[23]}$

Prevalence of TNBC in India is considerably higher compared with that seen in Western populations as suggested by various meta-analysis studies. Trial from India showed $51.2 \%$ HR positive tumours, HER2 positive tumours in $16.7 \%$ and TNBC in $29.8 \% .^{[12]}$

In this study, $30.7 \%$ patients had luminal subtype, $11.4 \%$ had triple positive, $13.7 \%$ had HER2 positive, $25.6 \%$ had triple negative and $17.5 \%$ had unknown status. The overall receptor expression pattern of patients in this study suggests a lower fraction of endocrine receptor-positive, higher fraction of triple negative and similar fraction of HER2positive disease compared to the Western data.

As reported by TMH India, this study also found a lower proportion of Indian breast cancer patients have hormone receptor positive and a higher fraction have triple negative phenotype compared to Western populations. These differences could, at least partly, be explained by the lower average age of our patients.

In this study significant proportion of patients $(31 \%)$ had co-morbid illness and were able to tolerate planned treatment similar to a patient without co-morbid illness. Thirteen percentages of women defaulted treatment with highest being in LABC (15.2\%) followed by early breast cancer $(9.8 \%)$.This reflects the lower socioeconomic status being the reason for treatment discontinuation.

Retrospective analysis of Southwest Oncology Studies reported 10 year DFS was $71 \%$ for African American patients and $78 \%$ for white patients; for OS, the 10-year estimates were $86 \%$ and $76 \%$, respectively. ${ }^{[24]}$

In this study with a median follow up of 45 months (28-64 months), 48 (15.2\%) patients in early breast cancer and 65 (21.9\%) patients in LABC died. The overall mean DFS and OS in early breast cancer was 57 months and 58 months respectively and locally advanced breast cancer was 51 months and 52.5 months respectively. In early breast cancer with luminal A had 3 year DFS and OS of $86 \%$ and $90 \%$ compared to TNBC with $70 \%$ and $60 \%$ respectively and patients with LABC with luminal A had 3 year DFS and OS of $82 \%$ and $80 \%$ compared to TNBC with $60 \%$ and $58 \%$ respectively which is comparable to Indian survival data.

The SEER data from National Cancer Institute showed 5 year survival as close to $100 \%, 93 \%, 72 \%$ and $22 \%$ in stage 0 or I, II, III and stage IV respectively. A trial from Tata Memorial Hospital (TMH) in patients with early breast cancer treated with multimodal treatment revealed 5 year disease free survival (DFS) rate of $70 \%$ and overall survival rate of $78 \%$. $^{[17]}$

Reina Haque et al reported, Women with luminal A tumors had the longest survival, although women with HER2enriched and luminal B tumors had much shorter survival times.Women with basal-like tumors had intermediate survival times, with deaths occurring earlier than women with luminal A tumors. Survival declined precipitously during the first 3 to 4 years of follow-up for both HER2 subtypes (HER2-enriched and luminal B), followed by a slowing in the decline over subsequent years of follow-up. The basal-like subtype showed a similar early decline over the first 2 to 2.5 years with a more gradual decline to about 13 years of followup. Interestingly, the curve for luminal A continues to decline steadily after 10 years of follow-up suggesting that the risk of late mortality persists in this group. ${ }^{[25]}$

\section{Conclusion}

Breast cancer is the most common cancer and the leading cause of cancer mortality among females. In this study, most of the patients presented a decade earlier and more advanced staged disease with higher percentage of TNBC and HER2 positive disease compared to western countries. Higher rate of defaulters for treatment especially in advanced stage could due poor socioeconomic support. The survival of patients was comparable with other Indian literature, but less than that of western data.

\section{References}

1. Yeole BB, Kurkure A (2003). An epidemiological assessment of increasing incidence and trends in breast cancer in Mumbai and other sites in India, during the last two decades. Asian Pac J Cancer Prev, 4, 51-6.

2. Pakseresht $\mathrm{S}$, Ingle $\mathrm{G}$, Bahadur A et al. Risk factors with breast cancer among women in Delhi. Indian J Cancer 2009; 46: 132-38.

3. Dey S, Boffetta P, Mathews A, Brennan P, Soliman A, Mathew A. Risk factors according to estrogen receptor status of breast cancer patients in Trivandrum, South India. Int J Cancer 2009; 125: 1663-70.

4. Gajalakshmi V, Mathew A, Brennan P et al. Breastfeeding and breast cancer risk in India: a multicenter case-control study. Int J Cancer 2009; 125: 662-5.

5. Nagrani RT, Budukh A, Koyande S, Panse NS, Mhatre SS, Badwe R. Rural urban differences in breast cancer in India. Indian J Cancer 2014; 51: 277-81.

6. Freddie Bray, Jacques Ferlay M, Isabelle Soerjomataram, Rebecca L. Siegel, Lindsey A. Torre, AhmedinJemal. Global cancer statistics 2018: GLOBOCAN estimates of incidence and mortality worldwide for 36 cancers in 185 countries. CA: A Cancer Journal for Clinicians 2018;0:1- 31 .

7. Anonymous. Three Year Report of Population Based Cancer Registries 2012-2014. Indian Council of Medical Research (ICMR), Bangalore, India 2016.

8. US Incidence of Breast Cancer Subtypes Defined by Joint Hormone Receptor and HER2 Status. Nadia Howlader, Sean F. Altekruse, Christopher I. Li, Vivien W. Chen, Christina A. Clarke, Lynn A. G. Ries, Kathleen A. Cronin Manuscript received June 26, 2013; revised November 22, 2013; accepted February 10, 2014.

9. Mathew A, Pandey M, Rajan B (2002) Do younger women with nonmetastatic and non-inflammatory breast carcinoma have poor prognosis? World J SurgOncol 2:2. 
10. Howlader N, Cronin KA, Kurian AW, Andridge Differences in Breast Cancer Survival by Molecular Subtypes in the United States. Cancer Epidemiol Biomarkers Prev. 2018 Jun;27(6):619-626.

11. Ghosh J, Gupta S, Desai S et al. Estrogen, progesterone and HER2 receptor expression in breast tumors of patients, and their usage of HER2-targeted therapy, in a tertiary care centre in India. Indian J Cancer 2011; 48: 391-6.

12. Sung H, Rosenberg PS, Hartman WQCM et al. Female Breast Cancer Incidence Among Asian and Western Populations: More Similar Than Expected. JNCI J Natl Cancer Inst (2015) 107(7): djv107

13. Badwe R, Hawaldar R, Parmar V et al. Single-injection depot progesterone before surgery and survival in women with operable breast cancer: a randomized controlled trial. J ClinOncol 2011; 29: 2845-2851.

14. Ferlay J, Soerjomataram I, Dikshit R et al. Cancer incidence and mortality worldwide: sources, methods and major patterns in GLOBOCAN 2012. Int J Cancer 2015; 136: E359-86.

15. Sandhu D, Sandhu S, Karwasra R, Marwah S. Profile of breast cancer patients at a tertiary care hospital in northIndia. Indian J Cancer 2010; 47: 16-22.

16. Leong SP, Shen ZZ, Liu TJ et al. Is breast cancer the same disease in Asian andWesterncountries?World J Surg 2010; 34: 2308-24.

17. SEER Cancer Statistics Review [Internet]. Altekruse SF, Kosary CL, Krapcho M, et al, editors. SEER Cancer Statistics Review 1975-2007. National Cancer Institute. Bethesda. based on November 2009 SEER data submission, posted to the SEER web site, 2010. [Last cited on 2010 Nov 26]. Availaible from: http://seer.cancer.gov/ csr/1975_2007/. [Last accessed on 2011 June 04].

18. Populationcommission.nic.in [Internet]. India: National Commission on Population, Government of India; c2000 [Last updated on 2003 Sep 11; cited on 2010 Nov 26]. Availaible from: http://populationcommission.nic.in/facts1.htm. [Last accessed on
2011June 04].

19. Rhodes A, Jasani B, Balaton AJ, Barnes DM, Miller KD. Frequency of oestrogen and progesterone receptor positivity by immunohistochemical analysis in 7016 breast carcinomas: Correlation with patient age, assay sensitivity, threshold value and mammographic screening. J ClinPathol 2000;53:688-96.

20. Dent R, Trudeau M, Pritchard KI, Hanna WM, Kahn HK, Sawka CA, et al. Triple-negative breast cancer: Clinical features and patterns of recurrence. Clin Cancer Res 2007;13:4429-34.

21. Saber Fallahpour, Tanya Navaneelan, Prithwish, AlessiaBorgo. Breast cancer survival by molecular subtype: a population based analysis of cancer registry data. CMAJ OPEN 2017, 5(3) E735

22. Mustapha Abubakar, Hyuna Sung, Devi BCR, Jennifer Guida, TiengSwee Tang, Ruth M. Pfeiffer and Xiaohong R. Yang Breast cancer risk factors, survival and recurrence, and tumor molecular subtype: analysis of 3012 women from an indigenous Asian population. Breast Cancer Research (2018) 20:114

23. Ambroise M, Ghosh M, Mallikarjuna VS, Kurian A. Immunohistochemical profile of breast cancer patients at a tertiary care hospital in South India. Asian Pac J Cancer Prev 2011;12:625-9.

24. Treatment Quality and Outcomes of African American Versus White Breast Cancer Patients: Retrospective Analysis of Southwest Oncology Studies S8814/S8897

25. Reina Haque, Syed A. Ahmed, Galina Inzhakova, Jiaxiao Shi, Chantal Avila, Jonathan Polikoff, Leslie Bernstein, Shelley M. Enger, and Michael F. Press. Impact of Breast Cancer Subtypes and Treatment on Survival: An Analysis Spanning Two Decades. Cancer Epidemiol Biomarkers Prev; 21(10) October 2012

Copyright: (C) the author(s), 2019. It is an open-access article distributed under the terms of the Creative Commons Attribution License (CC BY 4.0), which permits authors to retain ownership of the copyright for their content, and allow anyone to download, reuse, reprint, modify, distribute and/or copy the content as long as the original authors and source are cited.

How to cite this article: Vidhya R, Kalaichelvi K, Kumar SS, Raja G, Karthik R. Baseline Characteristics and Outcomes of Breast CancerRetrospective Analysis from a Tertiary Center in India.Asian J. Med. Res. 2019;8(2):MC04-MC08.

DOI: dx.doi.org/10.21276/ajmr.2019.8.2.MC2

Source of Support: Nil, Conflict of Interest: None declared. 